\title{
Dandy-Walker Syndrome Successfully Treated With Cystoperitoneal Shunting
}

\author{
-Case Report- \\ Tadao MIYAMORI, Takashi OKABE*, Takeshi HASEGAWA, Kenji TAKINAMI, \\ and Tetsuya MATSUMOTO
}

Departments of Neurosurgery and *Pediatrics, Toyama Municipal Hospital, Toyama

\begin{abstract}
A neonate presented with Dandy-Walker syndrome manifesting as a large posterior cranial fossa cyst, aplasia of the lower cerebellar vermis, and elevation of the confluence of the sinuses but without hydrocephalus. A cystoperitoneal shunt was placed at one month after birth. The cyst diminished in size, and marked development of the cerebellar hemispheres and descent of the confluence of sinuses were observed, but not vermis development. The primary pathology of Dandy-Walker syndrome is posterior cranial fossa cyst formation due to passage obstruction in the fourth ventricle exit area and aplasia of the lower cerebellar vermis. The first choice of treatment in patients with Dandy-Walker syndrome in whom the cerebral aqueduct is open is cystoperitoneal shunt surgery, regardless of the presence or absence of hydrocephalus.
\end{abstract}

Key words: Dandy-Walker syndrome, cystoperitoneal shunt, surgical treatment

\section{Introduction}

Dandy-Walker syndrome is characterized by a large posterior cranial fossa cyst, aplasia of the lower cerebellar vermis, and elevation of the confluence of sinuses, and is usually (77-99\% of cases) complicated by hydrocephalus, although this is not essential for the diagnosis of Dandy-Walker syndrome. ${ }^{1,5,9,12,13)}$ Treatment of Dandy-Walker syndrome is not standardized. The preferred method for cases complicated by hydrocephalus is placement of a ventriculoperitoneal shunt. ${ }^{1)}$ The complication of hydrocephalus generally $(80 \%$ of cases $)$ appears within 3 months of birth. ${ }^{5)}$ However, there is no established treatment for the period in which no hydrocephalus has developed."

Here we report a case of Dandy-Walker syndrome not complicated by hydrocephalus treated by early cystoperitoneal shunt surgery.

\section{Case Report}

A boy was born by natural cephalic delivery on September 13, 1996 at 40 weeks gestation. Weight at

Received August 7, 1998; Accepted July 1, 1999 birth was $3138 \mathrm{~g}$. Apgar scores were 5 at 1 minute after birth and 9 at 5 minutes after birth. Neonatal asphyxia was observed and the infant was admitted to the neonatal intensive care unit of our hospital. At that time, the muscle tonicity of the extremities was low and there was little movement of the extremities. Sucking power was also weak. Attacks of apnea were frequently observed. No protrusion of the anterior fontanel was observed. Computed tomography and magnetic resonance (MR) imaging showed aplasia of the lower cerebellar vermis, a large posterior cranial fossa cyst, and elevation of the confluence of sinuses (Figs. 1 left and 2 left). The diagnosis was Dandy-Walker syndrome with no complicating hydrocephalus. The large cyst in the posterior cranial fossa communicated to the fourth ventricle. The cyst protruded posteriorly and was judged to be treatable by placement of a cystoperitoneal shunt.

On October 14, 1996, cystoperitoneal shunt surgery was performed, using a Pudenz-Schulte medical shunting system with a median pressure valve. Following surgery, cerebrospinal fluid was observed leaking from the incision wound in the occipital region. Additional suturing of the skin did not stop the leakage. On November 26, 1996, surgery 


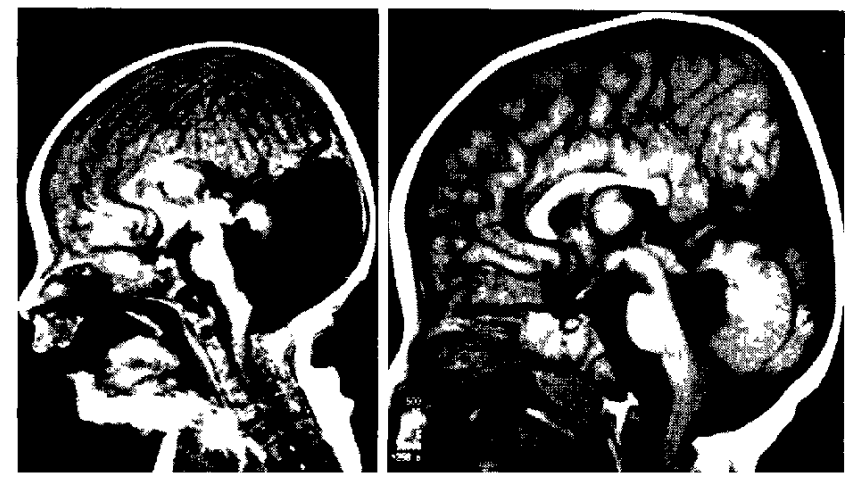

Fig. 1 Sagittal magnetic resonance images, preoperatively (left) showing hypoplasia of the vermis, a large cyst in the posterior cranial fossa, and elevation of the confluence of sinuses; and postoperatively (right) showing diminution of the cyst size and descent of the confluence of sinuses.

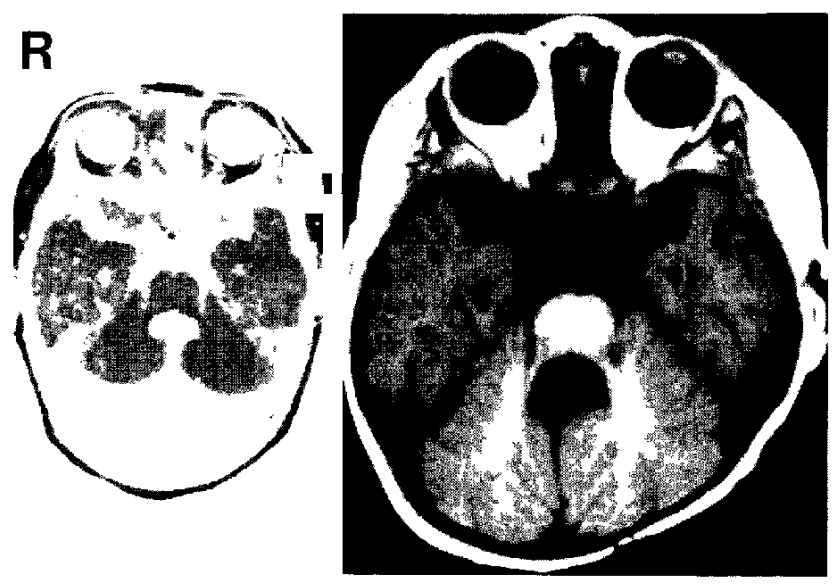

Fig. 2 Axial magnetic resonance images, preoperatively (left) showing a large cyst in the posterior cranial fossa and communicating to the fourth ventricle; and postoperatively (right) showing reduction in the cyst size and marked development of the cerebellar hemispheres.

to stop the cerebrospinal fluid leakage was performed. Adipose tissue taken from the abdomen was placed around the area where the shunt tube penetrated the dura, and then the area was covered with the fascia. The shunt valve was exchanged from median pressure to low pressure valve.

The cerebrospinal fluid leakage stopped after surgery; the action of the extremities increased and the episodes of apnea diminished in frequency. Postsurgical MR imaging on November 1997 revealed the cyst was reduced in size, and there was pronounced development of the cerebellar hemispheres and descent of the confluence of sinuses (Figs. 1 right and 2 right). No cerebellar vermis development was observed. Marked clinical improvement was observed from around July 1997. The patient showed movements of pursing the mouth. The muscle tonicity of the extremities greatly increased. However, this muscle tonicity has decreased from around July 1998. From around October 1997 , the neck was stable.

\section{Discussion}

The present patient presented with Dandy-Walker syndrome consisting of a large cyst communicating to the fourth ventricle in the posterior cranial fossa, aplasia of the lower cerebellar vermis, and elevated confluence of the sinuses without hydrocephalus. Post-surgical sagittal MR imaging showed the fourth ventricle appeared to be covered by the cerebellar vermis, but this is a part of the cerebellar hemisphere. Marked development of the cerebellar hemisphere was observed after placement of the shunt, but none of the cerebellar vermis. In this case, even after placement of the shunt, absolutely no development of the cerebellar vermis was observed. Aplasia of the lower cerebellar vermis is a primary, or essential, pathology of Dandy-Walker syndrome.

In contrast, descent of the confluence of sinuses was observed after placement of the shunt. In the axial section, the bone of the posterior cranial fossa projected backward before surgery and the projection alleviated after shunt. This indicates that the volume of the posterior cranial fossa reduced relatively. Although the tent ran on the straight upward posteriorly, at least the posterior region of the tent lowered in accordance with relative reduction of the volume of the fossa. After shunt, the cyst reduced and the bilateral cerebellar hemispheres grew markedly. Moreover, the confluence of sinus and the posterior region of the tent lowered and the volume of posterior cranial fossa reduced. Elevation of the confluence of the sinuses which occurs in DandyWalker syndrome may be due to prevention of telencephalic descent due to pressure caused by the posterior cranial fossa cyst, or more likely, mesodermal development damage prevents the descent of the tentorium cerebelli. ${ }^{33}$ However, after placement of the shunt, in our case, descent of the confluence of sinuses was associated with a reduction in the size of the cyst, which strongly supports the former. Elevation of the confluence of sinuses is thus a secondary pathology of the increase in posterior cranial fossa pressure due to the cyst in the posterior cranial fossa. 
In this case, marked development of the cerebellar hemispheres was observed 2 months after placement of the shunt, marked enhancement of muscle tonicity was observed 9 months after placement of the shunt, and the neck was stable 12 months after shunt surgery. Cerebellar development following shunt placement in patients with Dandy-Walker syndrome, if observed over an extended period of time, does not always lead to improved development of cerebellar function. ${ }^{4)}$ In general, decline of muscle tonicity in the acute stage occurs due to cerebellar hemisphere damage caused by cerebral apoplexy and other such conditions. Consequently, the development of the cerebellar hemisphere in this case was probably a factor in the improvement of muscle tonicity.

Dandy-Walker syndrome is generally treated by one of four methods: Excision of the epithelium by suboccipital craniotomy, ventriculoperitoneal shunt surgery, cystoperitoneal shunt surgery, or combined ventriculoperitoneal and cystoperitoneal shunt surgery. ${ }^{12]}$ The first method has a poor surgical record for children under the age of 2 or 3 years and is not generally applied., ${ }^{5,7,9]}$ Ventriculoperitoneal shunt surgery is often performed in cases complicated by hydrocephalus, ${ }^{1)}$ but the aqueduct constricts after ventriculoperitoneal shunt surgery. Therefore, the posterior cranial fossa cyst is not reduced and pressure in the posterior cranial fossa increases relatively, so cystoperitoneal shunt surgery may also be required. ${ }^{1,9)}$ Combined ventriculoperitoneal and cystoperitoneal shunts have always been used. ${ }^{12)}$ The preferred method of treatment may be cystoperitoneal shunt. ${ }^{2)}$ However, there is no standard treatment for Dandy-Walker syndrome complicated by hydrocephalus. ${ }^{6}$ )

Dandy-Walker syndrome may result from an unobstructed foramen of Luschka associated with an obstructed Magendie's foramen. ${ }^{5)}$ Hydrocephalus will eventually occur even if not present at the time of birth. Enlargement of the cyst may result in functional constriction of the aqueduct, which later causes hydrocephalus. In our patient, sagittal MR imaging revealed that the aqueduct was patent and passage through the aqueduct was thought to be satisfactory. We consider that treatment by cystoperitoneal shunt is adequate if the aqueduct is patent prior to surgery, even if hydrocephalus is a factor, and that ventriculoperitoneal shunt placement is unnecessary.

Complications following placement of a cystoperitoneal shunt include cerebrospinal fluid leakage after the shunt, as in this case; hematoma in the posterior cranial fossa; and brainstem symptoms associated with overflow of cerebrospinal fluid.
None of these complications are uncommon. ${ }^{7-11)}$ Such complications may be prevented by the use of a programmable valve for pressure control, although not used in this case.

\section{References}

1) Bindal AK, Storrs BB, Maclone DG: Management of the Dandy-Walker syndrome. Pediatr Neurosurg 16: 163-169, 1990

2) Domingo Z, Peter J: Midline developmental abnormalities of the posterior fossa: Correlation of classification with outcome. Pediatr Neurosurg 24: 111118, 1996

3) Gardner E, O'Rahilly R, Prolo D: The Dandy-Walker and Arnold-Chiari malformations: Clinical, developmental, and teratological considerations. Arch Neurol 32: 393-407, 1975

4) Gerszten PC, Albright AL: Relationship between cerebellar appearance and function in children with Dandy-Walker syndrome. Pediatr Neurosurg 23: 8692, 1995

5) Hirsch JF, Pierre-Kahn A, Renier D, Sainte-Rose C, Hoppe-Hirsch E: The Dandy-Walker malformation. J Neurosurg 61: 515-522, 1984

6) Kalidasan V, Carrol T, Allcutt D, Fitzgerald RJ: The Dandy-Walker syndrome - A 10-year experience of its management and outcome. Eur J Pediatr Surg 5 (Suppl 1): 16-18, 1995

7) Lipton HL, Preziosi TJ, Moses H: Adult onset of the Dandy-Walker syndrome. Arch Neurol 35: 672-674, 1978

8) Liu J, Ciacci JD, George TM: Brainstem tethering in Dandy-Walker syndrome: a complication of cystoperitoneal shunting: Case report. J Neurosurg 83: 1072-1074, 1995

9) Marinov M, Gabrovsky S, Undjian S: The DandyWalk syndrome: diagnostic and surgical considerations. Br J Neurosurg 5: 475-484, 1991

10) Mayhew JF, Miner ME, Denneny J: Upper airway obstruction following cyst-to-peritoneal shunt in a child with a Dandy-Walker cyst. Anesthesiology 62: 183-184, 1985

11) Naidich TP, Radkowski MA, Mclone DG, Leestma J: Chronic cerebral herniation in shunted DandyWalker malformation. Radiology 158: 431-434, 1986

12) Osenbach RK, Menezes AH: Diagnosis and management of the Dandy-Walker malformation: 30 years of experience. Pediatr Neurosurg 18: 179-189, 1992

13) Peterson DI, Stirling K, Pena AM: Dandy-Walker syndrome without hydrocephalus in an adult. Bull Clin Neurosci 48: 115-121, 1983

Address reprint requests to: T. Miyamori, M.D., Department of Neurosurgery, Toyama Municipal Hospital, 2-1 Imaizumi Hokubu-machi, Toyama 939-8075, Japan. 